\title{
Chest wall pain management after chest wall trauma
}

\author{
Federica Lovisari ${ }^{1}$, Martina Favarato ${ }^{2}$, Ilaria Giovannini ${ }^{1}$, Riccardo Giudici ${ }^{1}$, Roberto Fumagalli ${ }^{1,2}$ \\ ${ }^{1}$ Anesthesia and Intensive Care, ASST Niguarda, Milan, Italy; ${ }^{2}$ University of Milano Bicocca, Milan, Italy \\ Contributions: (I) Conception and design: All authors; (II) Administrative support: F Lovisari; (III) Provision of study materials or patients: F Lovisari, \\ M Favarato, M Favarato, R Giudici; (IV) Collection and assembly of data: F Lovisari, M Favarato, M Favarato; (V) Data analysis and interpretation: \\ F Lovisari, R Giudici, R Giudici; (VI) Manuscript writing: All authors; (VII) Final approval of manuscript: All authors. \\ Correspondence to: Federica Lovisari. Anesthesia and Intensive Care, ASST Niguarda, Milan, Italy. Email: federica.lovisari@gmail.com.
}

\begin{abstract}
Blunt chest trauma is defined as a high energy injury to the thorax and its structures, and it is usually a strong indicator of severe injury. Its clinical presentation can vary from rib fractures to pneumothorax, hemothorax, lung and soft tissue contusion; these lesions and consequential pain predispose to respiratory failure by compromising respiratory mechanics and by exacerbating pre-existing lung disease. This picture can lead to a number of complications, such as pneumonia or ARDS, that have a significant impact on patients' morbidity and mortality. One of the cornerstones of blunt chest trauma management is providing early and effective analgesia: pain control improves respiratory mechanics, reduces possible complications and allows effective and aggressive physiotherapy. In literature, there are few clinical trials comparing different analgesic techniques for pain relief in blunt thoracic trauma; our review aims to detail the clinical implication, adequacy and possible complications of different analgesic approaches commonly used in thoracic trauma management, such as epidural anesthesia, regional anesthesia, endovenous and intrathecal analgesia. This review is also focused on management and prevention of chronic pain. Pain management, lung toilet, aggressive respiratory therapy and early mobilization are key to a successful patient recovery. In selected patients, operative fixation may lead to better outcomes although further research is needed to clearly identify the category of patients and the ideal timing for surgical intervention. Chronic pain develops in up to $50 \%$ of the patients after a thoracic trauma. A multimodal approach tackling both its somatic and neuropathic component is essential to chronic pain prevention. Early and effective pain control demonstrated to have a pivotal role on patient recovery. While endovenous analgesia constitutes the method of choice for intensive care unit (ICU) sedated patients, regional unilateral analgesia should be considered in awake and spontaneously breathing patients. The approach of choice should be tailored on the single patient and, if possible, a multimodal strategy should be established.
\end{abstract}

Keywords: Thoracic trauma; regional anesthesia; multimodal analgesia; pain management

Received: 12 July 2019; Accepted: 28 October 2019; Published: 05 April 2020.

doi: 10.21037 /jovs.2019.11.01

View this article at: http://dx.doi.org/10.21037/jovs.2019.11.01

\section{Introduction}

Blunt chest trauma is defined by a high energy injury applied to thoracic structures; it is usually a consequence of motorcycle or car crashes, pedestrian investment or precipitation.

It may present with different clinical pictures: multiple rib fractures of both the anterior and posterior chest wall, flail chest, pneumothorax, hemothorax, sternal fractures, lung and soft tissue contusion.

These lesions can compromise respiratory mechanics and exacerbate underlying lung injury and pre-existing respiratory disease, predisposing to respiratory failure $(1,2)$.

Chest wall instability and important pain induce reduction of deep breath, functional residual capacity (FRC) and coughing, with secretion clearance impairment; pulmonary contusion can also develop as a consequence of trauma. 


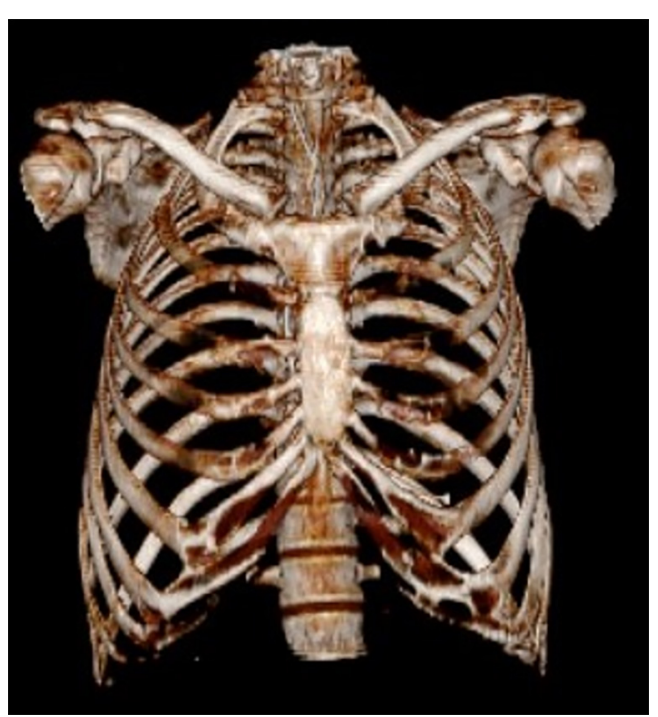

Figure 1 The bony thorax include the clavicles superiorly, the sternum and xyphoids anteriorly, twelve ribs bilaterally, the scapulaes and twelve vertebraes posteriorly.

Consequently, reduced lung compliance, ventilationperfusion mismatch, hypoxemia and hypercarbia and possible evolution in pneumonia and acute respiratory distress syndrome (ARDS) may develop.

One of the cornerstones of management is providing early and effective analgesia: pain control greatly ameliorates respiratory mechanics, reduces possible complications and allows effective and aggressive physiotherapy (1-3).

Thoracic injuries are a major source of morbidity and mortality in trauma patients, especially in blunt trauma; moreover, thoracic trauma is a strong indicator of severe injury and high trauma energy. Morbidity and mortality of blunt chest trauma increase with age and number of rib fractures $(1,4)$.

In literature, mortality rate of patients with blunt chest trauma hospitalized in intensive care unit (ICU) is $18 \%$ and can increase to $50 \%$ in case of complications $(1,5,6)$.

Chest trauma is also associated to cardiac and pulmonary contusion, aortic lesions and disruption, which increase either morbidity or mortality of patients (7).

Concomitant pulmonary dysfunction and need for prolonged mechanical ventilation, need for tracheostomy, subsequent wound and site infection, possible ventilationinduced lung injury and ventilator-acquired pneumonia, long ICU length of stay (LOS) and its complications all increase patient's morbidity and mortality $(1,2,8)$.

Not only acute pain and respiratory impairment, but also chronic pain, disability and quality of life impairment are possible consequences of blunt chest trauma $(1,4)$.

In literature, there are few clinical trials comparing different analgesic techniques for pain relief in blunt thoracic trauma; our review aims to detail the different analgesic approaches, their clinical implication, adequacy and possible complications.

\section{Anatomy}

The chest wall is a barrel-like structure, comprised by a bone-foundation overlaid by skin, soft tissue and musculature; all these structures contribute to respiratory function and mechanics (Figure 1).

Thoracic musculature is constituted by intercostal muscles and diaphragm, latissimus dorsi, serratus anterior and pectoralis major and minor muscles.

The anterior rami of the first 11 thoracic spinal nerves form the intercostal nerves, which pass along the inferior border of the rib with the vascular component (intercostal vein and artery).

This design provides strength, support and protection to the thoraco-abdominal viscera and allows for efficient volumetric changes in chest dimension, aiding in stabilizing the chest wall and, more importantly, participating in respiratory mechanics (9).

Given the considerable amount of energy required to determine a fracture in the chest wall bone compartment, this finding should always remind the clinician to look for an underlying thoracic or abdominal visceral injury. It should always be kept in mind that, in elderly people, less energy is required to provoke a fracture (4).

Given its anatomy, when the rib is damaged by a blunt chest trauma, visceral, neural and vascular lesions may occur (Figures 2,3).

Alteration to the skeletal formation of the thoracic outlet can cause neurovascular impingement, resulting in thoracic outlet syndrome.

The main symptoms of thoracic outlet syndrome are pain, numbness, weakness and paraesthesia of the upper limbs, provoked by the lesion of the brachial plexus and, if the subclavian artery is consequently compressed or damaged, it can also induce ipsilateral limb ischemia.

This has to be recognized and treated promptly in order to preserve the neural function as well as the vitality of the limb.

Respiratory function can be seriously affected by chest trauma: a lung contusion is frequently associated, moreover, 


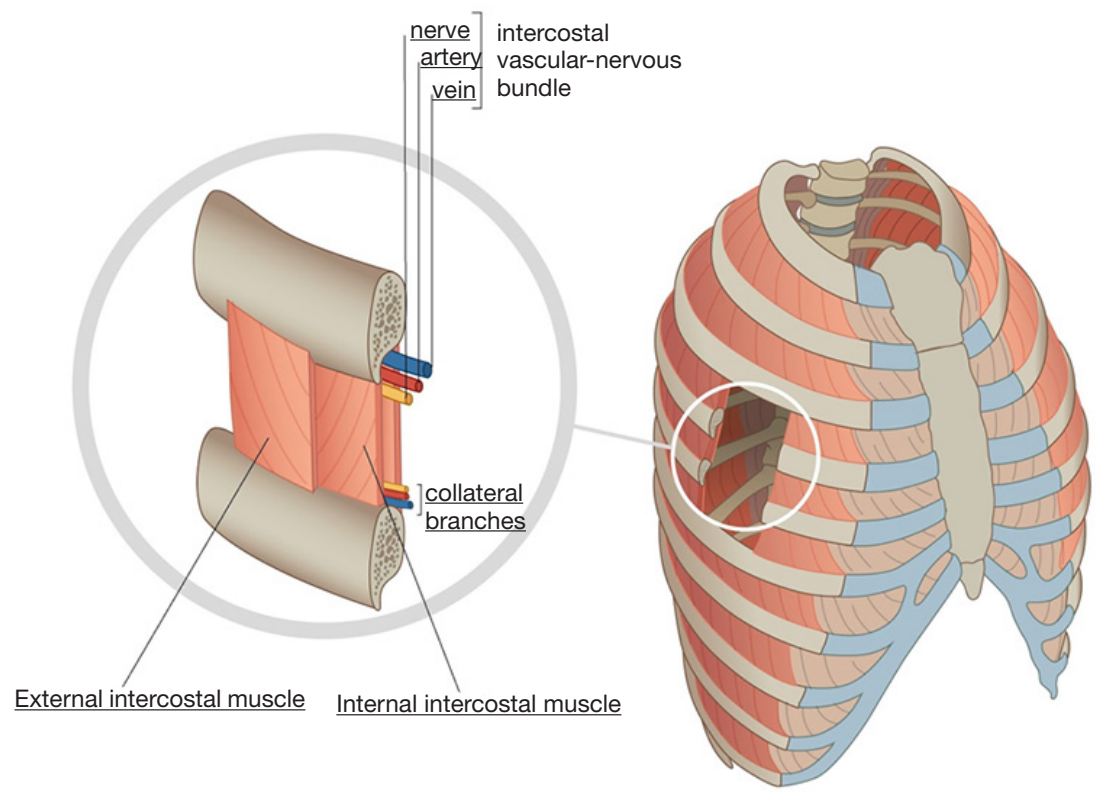

Figure 2 In this picture it is shown how close is the vascular-nervous bundle to the rib. It is easily understood why a fracture of this bone can cause a lesion in adjacent structures.

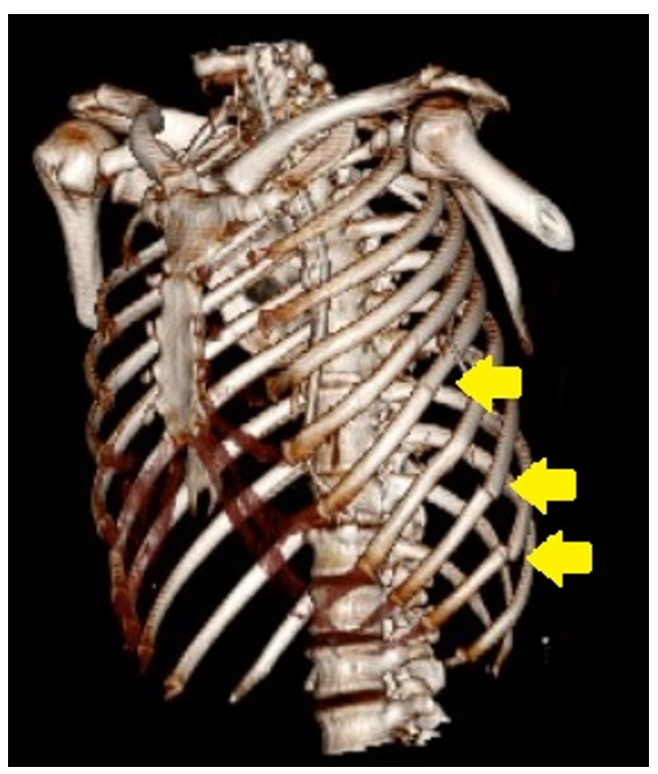

Figure 3 A 3D reconstruction of a thoracic CT scan of a blunt chest trauma (car accident with lateral impact), showing multiple rib fractures (indicated by arrows).

hemorrhage or edema from energy transfer or shear-type forces at the inertial interfaces may cause hypoxemia and an increase of arterio-venous shunting.

Moreover, in the severely injured patient, we cannot forget the effects of the damage control resuscitation, such as aggressive correction of hypovolemia, acidosis, hypothermia, and coagulopathy, that may also play a role in the development of significant complications. Fluid administration must be carefully delivered to avoid lung edema and worsening of pulmonary contusions (9).

Another cause of respiratory impairment is due to the possible effect of pain: it reduces the ability to take deep breaths and cough, with a decrease in tidal and lung volume. In addition, bronchial secretions can cause atelectasis and alveolar collapse, further worsening respiratory exchange.

In a recent preliminary study published in 2018 , it is suggested that, in emergency settings, a serial evaluation of forced vital capacity (FVC) could be a reliable marker of trauma severity as well as a reliable method to assess the effectiveness of its management: a persistently low FVC might in fact advise against ward admission and advocate for more aggressive treatment, especially in patients with increased risk of complications (10).

All of these factors can influence patient's morbidity and mortality, as well as their ICU and hospital LOS (5).

\section{Chest pain components}

Chest pain comprehends musculoskeletal or somatic pain and referred pain from the viscera (heart, lung, oesophagus), 
or visceral pain.

The differential diagnosis, in some cases, becomes an arduous task: both somatic and visceral pain belong to the nociceptive pain spectrum in which the information is sent to and elaborated by the central nervous system (CNS) as a consequence of the stimulation of pain receptors by a noxious stimulus (mechanical, thermical or chemical) that has caused tissue damage.

Musculoskeletal pain is easily localized; the higher number of receptors with a small receptive field enhances the discriminatory capacity when the information is elaborated by the CNS.

Pain receptors are also found in most of thoracic, abdominal and pelvic organs and its surrounding connective tissue, but their receptive field is wider with a therefore lower discriminatory capacity. These receptors are responsible for visceral pain which, is often difficult to allocate to a specific organ.

In fact, the nociceptive information is channelled by C-fibers: unmyelinated afferent fibers directed to many central neurons in the spinal cord, that also receives information from somatic fibers.

All inputs are then processed by the cortex: when the noxious input is combined with a somatic one, it often produces a poorly localized pain response. However, damage to the organ capsule or direct injury to deep tissue may be more easily localized (11).

In addition to somatic and visceral pain, it is of great importance the recognition and treatment of neuropathic pain. This particular type of pain is frequently follows a lesion situated at any level of the nervous system (central and/or peripheral) and determined by the trauma itself or by its treatment (surgery or drugs-receptors interactions).

Neuropathic pain typically occurs in the area of the primary injury and it then broadens to the correspondent territory of the nerve involved.

The intensity of pain ranges from moderate to severe and it is often described as a burning or shooting sensation. Hyperalgesia and allodynia are common features of this type of pain.

The injury is followed by a cytokine inflammatory cascade that produces an activation and/or a sensitization of nociceptors. Attempts of healing lead to a disorganized sprouting of nerve fibers, responsible for the increasing nociceptive input, even from innocuous stimuli.

Another important feature is the "wind-up pain": a neuroplastic phenomenon determined by a frequencydependent increase in the excitability of spinal cord neurons.
This event leads to an amplification of the nociceptive signal that arrives to the spinal cord from peripheral nociceptors connected to C-fibers (12).

The diagnosis of neuropathic pain is not always easy to accomplish but it must always be considered especially when pain does not respond to first-line analgesia. Drugs such as neuroleptics, anti-depressants and anti-epileptics may be required.

\section{Management}

Immediate chest trauma treatment can be conservative (with invasive or non invasive mechanical ventilation, aggressive respiratory and pain therapy, lung toilet) or operative (surgical internal fixation or open reduction of flail chest) (1,4-6).

Conservative treatment is usually the treatment of choice; it consists of tracheal intubation (with or without subsequent tracheostomy) and invasive mechanical ventilation for more injured patients, or non-invasive mechanical ventilation in patients who are less injured.

Both invasive and non-invasive mechanical ventilation can be challenging in blunt chest trauma patients and require ICU admission.

Often, the presence of pneumothorax and/or hemothorax pose the need for chest drainage and complicates the ventilatory approach, with challenge in end expiratory, mean airway and peak pressure level selection, also making ventilation and recruitment of atelectatic and contused part of the lung complicated.

Lung toilet, aggressive respiratory therapy and early mobilization are key to success in patient recovery.

Nonetheless, in selected patients, operative fixation may lead to better outcomes, even though nowadays only less than $1 \%$ of patients undergo surgical stabilization after blunt chest trauma.

Literature is not clear about surgical fixation indication and techniques; case-reports and randomized controlled trials compare a wide variety of different pathologies (from unicortical non-displaced fracture in stable patients to severely displaced fractures in flail chest) and approaches (percutaneous thoracoscopic with muscle sparing versus open thoracotomy).

Generally, ribs 1-2, 11-12 are not considered for surgical stabilization because of their minimal contribution to chest wall integrity; ribs 3-10 should, on the other hand, be considered for stabilization in patients with flail chest.

Further research is needed to clearly define the category of patients who will benefit most from surgical 
stabilization and to identify the ideal timing for surgical intervention $(1,4,6)$.

\section{Analgesia (Table 1)}

\section{Epidural analgesia (EA)}

EA is extremely effective in acute pain treatment; it ameliorates pulmonary function test (FRC, lung compliance, vital capacity, airway resistance reduction and respiratory exchange amelioration) and patient participation to physiotherapy with an increase number of deep breathing and a more effective cough.

EA is of level I recommendations in blunt chest trauma patients, either with lumbar or thoracic approach depending on lesion localization; in patients with bilateral rib fractures $\mathrm{EA}$ is the technique of choice.

Usually, local anesthetic (LA) with or without adjuvants (opioids, clonidine) are administered in boluses or as continuous infusion $(2,8,13)$.

Pain relief with EA is dramatic, immediate and superior to either intravenous (IV) and intra-pleural block; a reduction in patient's inflammatory response, with a decrease in interleukin-8 secretion, has also been demonstrated $(1,3)$.

In patients older than 60 years old, EA decreases mortality and incidence of pulmonary complication; in all patients, it shortened ICU and hospital LOS $(1,3,4)$.

EA is contra-indicated in coagulopathic patients or in patients with elevated clotting time, vertebral fractures, acute spinal cord injuries and sepsis; it can be difficult and technically demanding, especially in distressed patients.

EA can mask abdominal injuries and enhance hypotension in polytraumatized hypovolemic patients for its sympatholytic action; rare complications are epidural infections and dural puncture (2,3,6,8,13-16) (Video 1).

\section{Regional analgesia}

The second possible choice for pain management is regional anesthesia: paravertebral nerve block (PVB), intercostal nerve block (ICNB) and serratus plane block with LA achieve a level of analgesia comparable to EA, but with fewer side effects and complications $(6,14,15)$.

Less diffused but described in literature are subrhomboidal block and intrapleural analgesia (1).

Lack of systemic sedation, relative low incidence of hypotension and a lower technical complexity when compared to EA are the main advantages of loco-regional approaches; nonetheless, relative contraindication are coagulopathic patients, risk of LA toxicity and the possibility of local site infection (13).

\section{PVB}

PVB blocks the posterior primary branch of the ipsilateral sympathetic and somatic chain by anesthetizing spinal nerves, pre and postganglionic sympathetic nerves; it is an extremely effective method for pain control, especially in unilateral multiple rib fractures.

In literature, PVB is as effective as EA in pain control $(1,2,8,13,16)$.

PVB allows optimal physiotherapy, as demonstrated by the clear amelioration of respiratory functional tests, respiratory performance, mechanics and oxygenation.

It can be use either with a single injection or a catheter positioning and it does not require costal palpation, which is extremely uncomfortable in patients with intense pain.

Easier to perform than thoracic EA, it can also be performed in patients heavily sedated or with a low Glasgow Coma Scale because of the absent danger of spinal cord injury and a very low incidence of hypotension because of the unilateral sympathetic block $(1,2,8,13,16)$.

PVB is relatively contraindicated in coagulopathic patients or in patients with elevated clotting time; possible complications are hypotension, vascular and pleural puncture and pneumothorax. Inadvertent EA and intrathecal injection have also been described (2,13-15).

\section{ICNB}

ICNB is most effective when it involves the intercostal nerve proximal to the fractured side.

Nonetheless, because of the overlapping innervation from segments above and below ribs, it is necessary to block the nerve either above and below the fractured site $(3,13)$.

A block posterior to the mid-axillary line will ensure blockade of either the lateral cutaneous or the anterior branch of the intercostal nerve.

Amelioration of pulmonary function test, decrease of IV and oral opioids consumption and decrease hospital LOS have been well demonstrated with ICNB; nonetheless, at present, evidence to support its use is still limited $(1,4,8)$.

ICNB is relatively contraindicated in coagulopathic patients or in patients with elevated clotting time; because of the necessity of multiple injections, it is also more prone to possible LA toxicity and incidence of pneumothorax than other analgesic solutions.

Moreover, in multiple rib fractures, it is a source of 


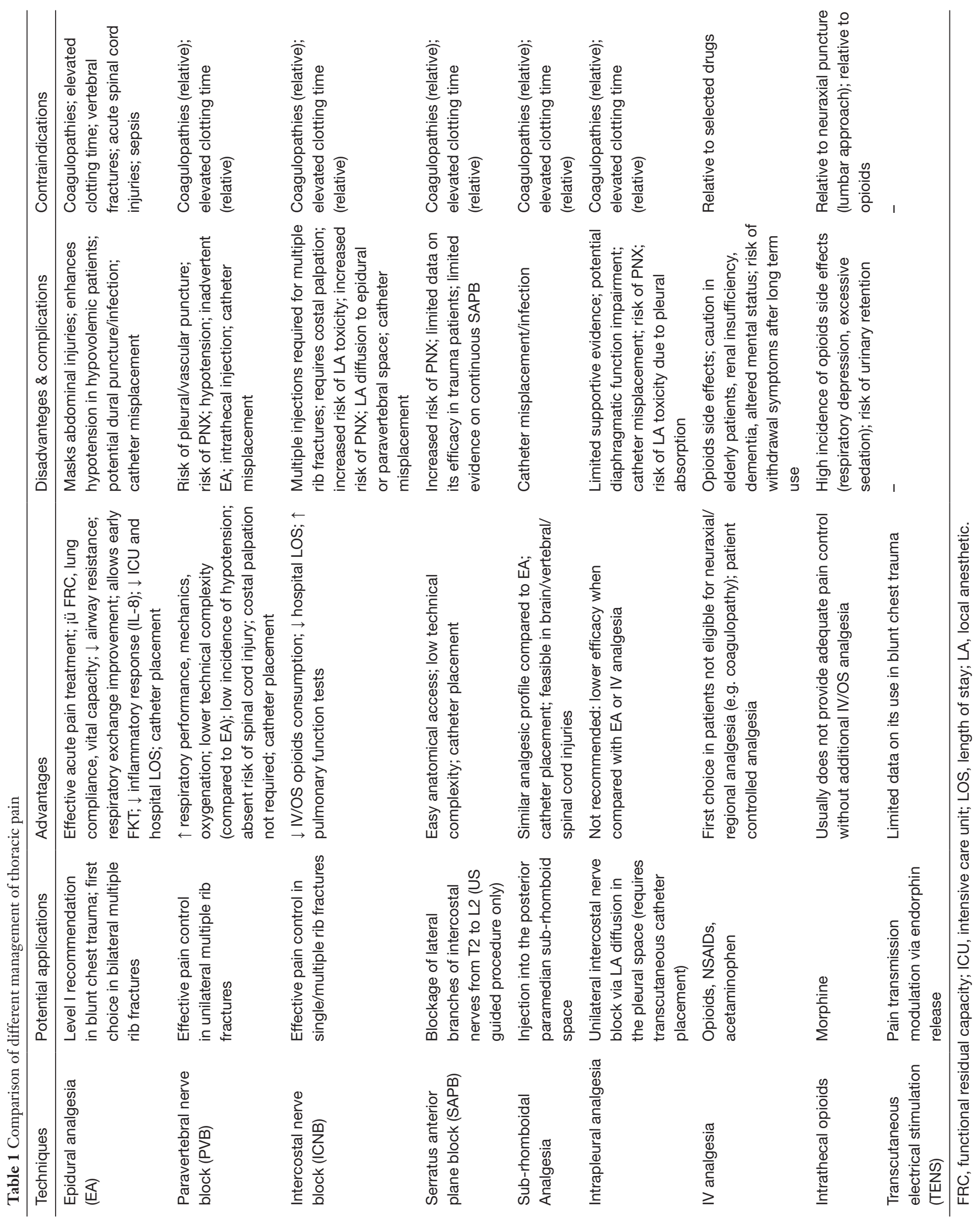




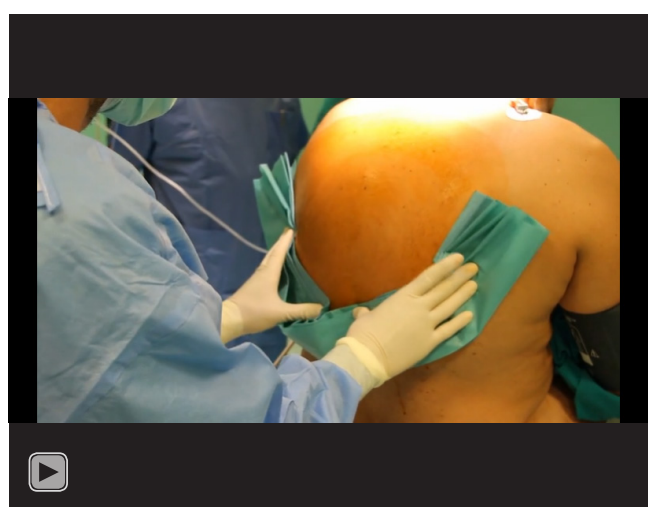

Video 1 Procedure of a peridural catheter positioning (17).

discomfort for patients because of the need of fractured costal palpation. It is also extremely time-consuming, considering that it requires the repetition of this technique for each fractured rib $(3,13)$.

Problems of continuous ICNB with transcutaneous catheter positioning are misplacing of the catheter (too close to subcutaneous space or to deep into the intrapleural space) or inadverted LA diffusion to either the epiduralor para-vertebral space or a combination of the two spaces $(3,14)$.

\section{Serratus plane block}

Serratus plane block provides analgesia to the thorax by blocking the lateral branches of the intercostal nerves from T2 to L2.

It consists of the blockage of the nerve branches between the anterior serratus and the latissimus dorsi muscle.

To date, few case reports can be found in literature regarding this approach in trauma patients, but its feasibility and easy anatomical access are promising for unilateral analgesia in blunt chest trauma management $(15,18)$.

\section{Sub-rhomboidal analgesia}

This approach consists of LA injection into the posterior paramedian sub-rhomboid space.

In can be approached as single injection of LA or with catheter placement (with or without tunnelization for catheter stabilization).

This approach, when compared to EA, showed a similar analgesic profile; moreover, it can be used in patients with either brain trauma, vertebral and spinal cord injury $(1,16)$.

\section{Intrapleural analgesia}

Intrapleural analgesia has been proposed, via a transcutaneous catheter placement, to obtain unilateral ICNB through LA diffusion in the pleural space.

The patient needs to be supine for LA diffusion and multiple unilateral dermatomes block, which is not optimal in a patient with compromised respiratory mechanics; moreover, with this approach, there is a possibility of diaphragmatic function compromise with respiratory function worsening.

Possible side effects are intraparenchymal or extrapleural catheter placement, pneumothorax and chest wall misplacement; moreover, high plasma concentration via pleural absorption has been demonstrated, with a high potential for possible LA systemic toxicity $(1,3,8,13)$. Horner's Syndrome, pleural infections and catheter rupture have also been described (19).

Its use is limited and not strongly supported in literature; patients always reported lower pain thresholds and less analgesic use with EA or IV analgesia when compared to intrapleural analgesia $(1,8,13)$.

\section{Ultrasound-guided (US) block and catheter placement} US-guided block and catheter placement are a relatively new technique that is safer if compared to the solely landmark-based techniques.

The needle and different structures visualization (perinervous and paravertebral spaces, lungs, pleura and vascular structures) decreases complication rates (pneumothorax and intra-vascular LA injection); moreover, the echographic window allows to confirm the LA spread in the peri-nervous or para-vertebral space $(3,13,18)$.

A percutaneous US-guided catheter insertion allows for prolonged LA administration with intermittent boluses or continuous infusion, therefore reducing the need of additional IV/OS opioids and NSAIDs administration, improving mobilization and avoiding the necessity of multiple punctures (20).

\section{IV analgesia}

In patients not eligible for neuraxial or regional techniques, usually because of coagulation pathology (either intrinsic or iatrogenic), IV analgesia with opioids (with or without patient controlled analgesia) supported by non-steroidal anti-inflammatory drugs (NSAIDs) and acetaminophen is a widespread choice $(8,13)$.

Clearly, this is not the first choice for older patients, patients with an altered mental status (either because of dementia, Alzheimer, delirium or brain trauma), patients 
with renal insufficiency and in need for strict neurologic monitoring $(1,3,15,18)$.

Moreover, systemic side effects associated with prolonged and/or high dosage use of opioids should be kept in mind by the clinician: excessive sedation and respiratory depression may have a significant impact on patient's recovery, ICU and hospital LOS (21).

When necessary and possible, IV analgesia can be associated by intra-muscular, trans-dermal or oral routes (1).

\section{Intrathecal opioids}

Intrathecal opioids, especially morphine, have been used to control pain via lumbar approach. Usually this regimen is not enough by itself for adequate pain control, necessitating either multiple punctures or additional IV or oral doses.

A high incidence of opioids side effects has also been noted, with excessive sedation and respiratory depression worsening ventilatory impairment; nausea and urinary retention are other possible complications (3).

\section{Transcutaneous electrical stimulation (TENS)}

There are few data about the use of TENS in blunt chest trauma for pain treatment. It efficacy is based on endorphins release in the spinal cord with pain transmission modulation (3).

\section{Chronic pain}

The impact on chronic pain of different strategy for pain management is not clearly stated (6).

Chronic pain can be encountered in up to $50 \%$ of patients, with a significant impairment of life quality; loss of working force, high health-care utilization, depression and analgesic misuse are possible consequences of chronic pain development.

High standard and multimodal acute pain treatment, either in its somatic and neuropathic component, is the key to chronic pain prevention.

Chronic pain has three different etiologies: costal, pleural and neuropathic component.

No study has ever explored the impact of different acute analgesic approaches on chronic pain development and on its several etiologies.

To date, it seems that either loco-regional analgesia and a strong aggression of the neuropathic pain component with neuroleptics (pregabalin/gabapentin) are associated to a reduction in chronic pain development $(1,8,22)$.

\section{Conclusions}

Pain treatment in patients with blunt chest trauma, especially with respiratory compromise, should be instituted early and promptly.

The approach of choice should be individualized on the single patient, his comorbidities and clinical picture.

In ICU sedated and ventilated patients, IV analgesia is probably the best choice that also helps in multimodal sedation; in these patients, EA is contraindicated for possible masked complications and hypotension.

Regional unilateral analgesia is the best choice in awake, spontaneously breathing patients with unilateral fractures, while EA is indicated in multiple bilateral rib fractures.

Intrathecal opioids and TENS are rarely used in the trauma setting, both for inadequate analgesia level and for the numerous side effects of intrathecal opioids.

To date, no form of analgesia delivery is demonstrated to be superior to others in acute pain control and chronic pain development; nonetheless, a multimodal approach has been demonstrated to be effective in pain control and reduction of drugs side effects.

\section{Acknowledgments}

Funding: None.

\section{Footnote}

Provenance and Peer Review: This article was commissioned by the Guest Editors (Marco Scarci and Fabrizio Minervini) for the series "Chest Wall Traumas" published in Fournal of Visualized Surgery. The article has undergone external peer review.

Conflicts of Interest: All authors have completed the ICMJE uniform disclosure form (available at https://jovs. amegroups.com/article/view/10.21037/jovs.2019.11.01/ coif). The series "Chest Wall Traumas" was commissioned by the editorial office without any funding or sponsorship. The authors have no other conflicts of interest to declare.

Ethical Statement: The authors are accountable for all aspects of the work in ensuring that questions related to the accuracy or integrity of any part of the work are appropriately investigated and resolved. All procedures performed in this study were in accordance with the Helsinki Declaration (as revised in 2013). The manuscript 
is waived from patient informed consent according to the ethics committee or institutional review board.

Open Access Statement: This is an Open Access article distributed in accordance with the Creative Commons Attribution-NonCommercial-NoDerivs 4.0 International License (CC BY-NC-ND 4.0), which permits the noncommercial replication and distribution of the article with the strict proviso that no changes or edits are made and the original work is properly cited (including links to both the formal publication through the relevant DOI and the license). See: https://creativecommons.org/licenses/by-nc-nd/4.0/.

\section{References}

1. Kourouche S, Buckley T, Munroe B, et al. Development of a blunt chest injury care bundle: An integrative review. Injury 2018;49:1008-23.

2. Malekpour M, Hashmi A, Dove J, et al. Analgesic Choice in Management of Rib Fractures: Paravertebral Block or Epidural Analgesia? Anesth Analg 2017;124:1906-11.

3. Karmakar MK, Ho AMH. Acute Pain Management of Patients with Multiple Fractured Ribs. J Trauma 2003;54:615-25.

4. Majercik S, Pieracci FM. Chest Wall Trauma. Thorac Surg Clin 2017;27:113-21.

5. Michelitsch C, Acklin YP, Hassig G, et al. Operative Stabilization of Chest Wall Trauma: Single-Center Report of Initial Management and Long-Term Outcome. World J Surg 2018;42:3918-26.

6. Golic DA, Svraka D, Keleman N, et al. Epidural Analgesia With Surgical Stabilization of Flail Chest Following Blunt Thoracic Trauma in Patients With Multiple Trauma. Front Med (Lausanne) 2018;5:280.

7. Lee WJ, Lin HY. Severe chest pain after blunt chest trauma. Emerg Med J 2011;28:451-2.

8. Galvagno SM, Smith CE, Varon AJ, et al. Pain management for blunt thoracic trauma: A joint practice management guideline from the Eastern Association for the Surgery of Trauma and Trauma Anesthesiology Society. J Trauma Acute Care Surg 2016;81:936-51.

9. Saillant NN, Sein V. Management of severe chest wall trauma. J Emerg Crit Care Med 2018;2:41.</jrn>

10. Carrie C, Stecken L, Scotto M, et al. Forced Vital Capacity assessment for risk stratification of blunt chest trauma patients in emergency settings: a preliminary study. Anaesth Crit Care Pain Med 2018;37:67-71.

11. <eref $>11$. Oken JE, Hanyu-Deutmeyer A. Chest wall pain syndrome. PM\&R Knowledge NOW Originally published:09/20/2013; Last updated:7/28/2017. Available online: https://now.aapmr.org/chest-wall-painsyndrome/</erefs

12. Herrero JF, Laird JM, López-García JA. Wind-up of spinal cord neurons and pain sensation: much ado about something? Prog Neurobiol 2000;61:169-203.

13. Ho AM, Karmakar MK, Critchley LA. Acute pain management of patients with multiple fractured ribs: a focus on regional techniques. Curr Opin Crit Care 2011;17:323-7.

14. Murata H, Salviz EA, Chen S, et al. Ultrasound-Guided Continuous Thoracic Paravertebral Block for Outpatient Acute Pain Management of Multilevel Unilateral Rib Fractures. Anesth Analg 2013;116:255-7.

15. Fu P, Weyker PD, Webb CAJ. Case Report of Serratus Plane Catheter for Pain Management in a Patient With Multiple Rib Fractures and an Inferior Scapular Fracture. A A Case Rep 2017;8:132-5.

16. Shelley CL, Berry S, Howard J, et al. Posterior paramedian subrhomboidal analgesia vs thoracic epidural analgesia for pain control in patients with multiple rib fractures. J Trauma Acute Care Surg 2016;81:463-7.

17. Lovisari F, Favarato M, Giovannini I, et al. Procedure of a peridural catheter positioning. Asvide 2020;7:042. Available online: http://www.asvide.com/watch/33078

18. Fusco P, Scimia P, Di Carlo S, et al. Ultrasound-Guided Serratus Plane Block and Fast-Track Tracheal Extubation in the Operating Room for Thoracic Trauma Patients: A Case Report. A A Case Rep 2017;9:305-7.

19. Strømskag KE, Minor B, Steen PA. Side effects and complications related to interpleural analgesia: an update. Acta Anaesthesiol Scand 1990;34:473-7.

20. Ilfeld BM. Continuous Peripheral Nerve Blocks: An Update of the Published Evidence and Comparison With Novel, Alternative Analgesic Modalities. Anesth Analg 2017;124:308-35.

21. Benyamin R, Trescot AM, Datta S, et al. Opioid complications and side effects. Pain Physician 2008;11:S105-20.

22. Carrie C, Guemmar Y, Cottenceau V, et al. Long-term disability after blunt chest trauma: Don't miss chronic neuropathic pain! Injury 2019;50:113-8.

\section{doi: 10.21037/jovs.2019.11.01}

Cite this article as: Lovisari F, Favarato M, Giovannini I, Giudici R, Fumagalli R. Chest wall pain management after chest wall trauma. J Vis Surg 2020;6:18. 